\title{
Characterization of starch in Cucurbita moschata germplasms throughout fruit development
}

Hafiz Muhammad Khalid Abbas, He-Xun Huang, Yu-Fei Yang, Yuan-Heng Xie, Jian-Feng Zou, Shu-Dan Xue, Dong-Guang Song, Ting-Quan Wu, Jun-Xing Li and Yu-Juan Zhong*

Table S1. List of primers used for qRT-PCR.

\begin{tabular}{|c|l|}
\hline Gene & \multicolumn{1}{|c|}{ Primer sequence (5'-3') } \\
\hline Actin- $F$ & CGGCCATTGAGAAAAGCTACGAACT \\
\hline Actin- $R$ & CCCACCACTGAGGACGATGTTACCA \\
\hline$P G M-\mathrm{F}$ & GCTGGAGCAACTGTAAGAA \\
\hline$P G M-\mathrm{R}$ & GGACAAACATAATGCCAAA \\
\hline AGPase-F & ATCACTGTAGCTGCATTACCC \\
\hline AGPase-R & TCAACCTTCATCGCTTTCA \\
\hline$S S-\mathrm{F}$ & GGAATGGCAGGTAGGTT \\
\hline$S S-\mathrm{R}$ & ATCGTCCGCAGAGTTAT \\
\hline$G B S S-\mathrm{F}$ & TTGCAGGACTGCCAGTAGGA \\
\hline$G B S S-\mathrm{R}$ & AGAACATCACCAAGCCCAC \\
\hline$S B E-\mathrm{F}$ & TGTTGAAAGAGGGATTGCG \\
\hline$S B E-\mathrm{R}$ & TCATAACTCCACCCGTTGC \\
\hline$D B E-\mathrm{F}$ & GACTCGCACATCACCACATC \\
\hline$D B E-\mathrm{R}$ & TTTCATTGTATGGGCGGCTG \\
\hline
\end{tabular}




\begin{tabular}{|c|l|}
\hline$S P-\mathrm{F}$ & GGGCCACTACAGTTGGTTTG \\
\hline$S P-\mathrm{R}$ & TCTCCACATTTGCCCCATCT \\
\hline
\end{tabular}

Table S2. Genes involved in starch metabolism.

\begin{tabular}{|c|l|}
\hline Gene abbreviation & \multicolumn{1}{|c|}{ Unigene ID } \\
\hline$P G M$ & Unigene0002629, Unigene0037284 \\
\hline AGPase & $\begin{array}{l}\text { Unigene0047165, Unigene0040458, Unigene0040454, Unigene0039030, } \\
\text { Unigene0031387, Unigene0016505, Unigene0003314 }\end{array}$ \\
\hline GBSS & $\begin{array}{l}\text { Unigene0046257, Unigene0043814, Unigene0035250, Unigene0035248, } \\
\text { Unigene0029421 }\end{array}$ \\
\hline$S B E$ & $\begin{array}{l}\text { Unigene0023284, Unigene0038340, Unigene0038341, Unigene0052676, } \\
\text { Unigene0004574, Unigene0038339, Unigene0001527, Unigene0022306 }\end{array}$ \\
\hline$S S$ & $\begin{array}{l}\text { Unigene0001083, Unigene0007832, Unigene0021341, Unigene0021342, } \\
\text { Unigene0040073, Unigene0040074, Unigene0040075 }\end{array}$ \\
\hline$D B E$ & Unigene0053977, Unigene0027020, Unigene0032724, Unigene0032725 \\
\hline$S P$ & $\begin{array}{l}\text { Unigene0008725, Unigene0008726, Unigene0011332, Unigene0011333, } \\
\text { Unigene0012033, Unigene0012034, Unigene0014537, Unigene0015199, } \\
\text { Unigene0024594, Unigene0043760, Unigene0043761, Unigene0008725 }\end{array}$ \\
\hline
\end{tabular}


A

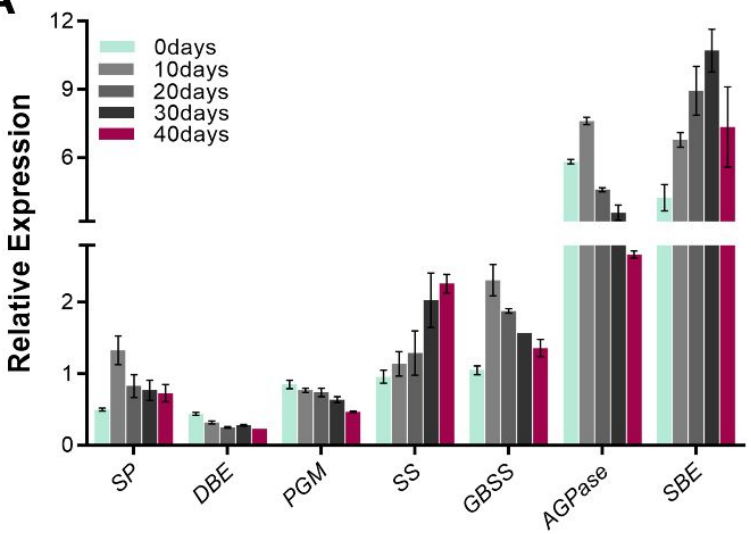

Starch Pathway Genes

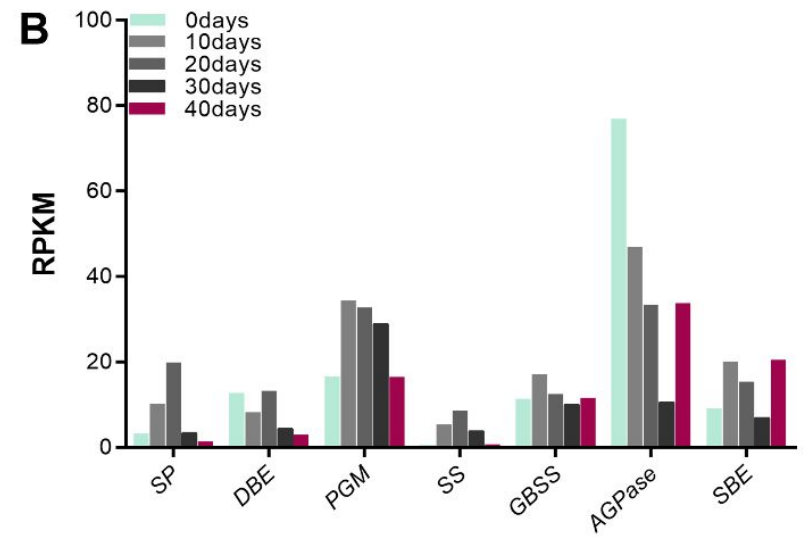

Starch Pathway Genes
C

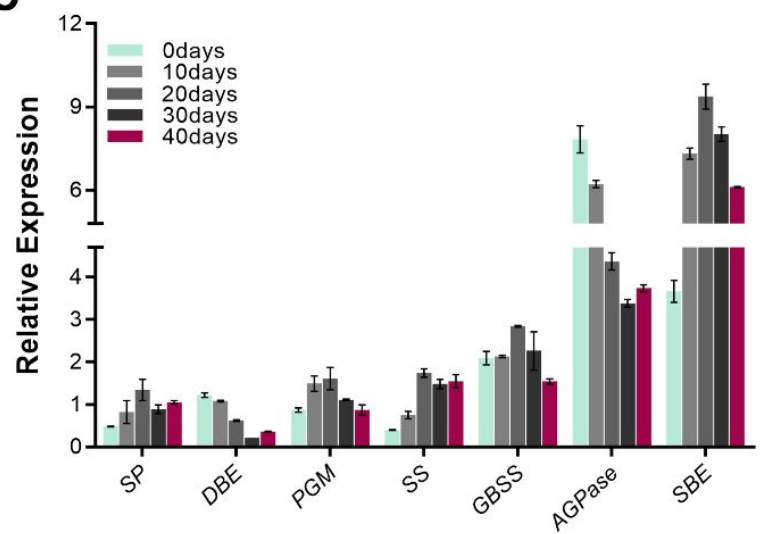

Starch Pathway Genes

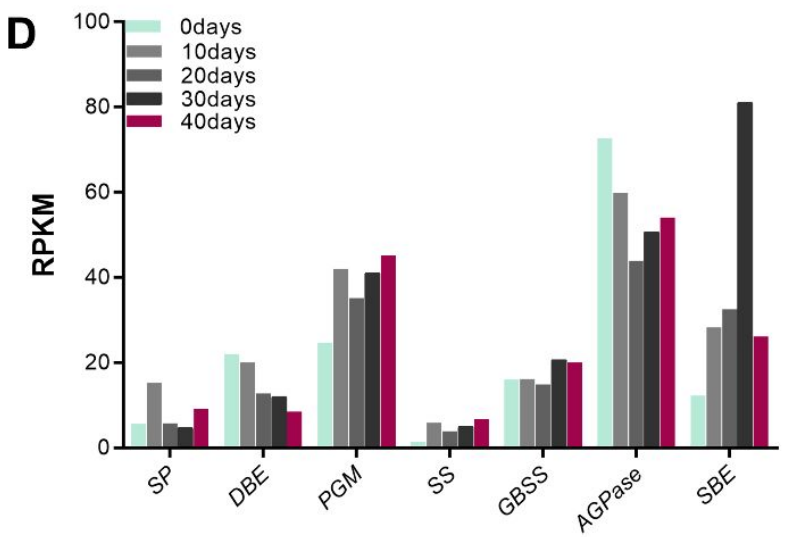

Starch Pathway Genes

Figure S1. Relative expression and RPKM of different genes from starch pathway.

Samples were collected at different stages of fruit development. RNA was extracted and cDNA was synthesized for qRT-PCR. Actin was used as internal control. (A) Relative expression of genes from starch pathways in CMO-E, (B) RPKM values of genes from starch pathways in CMO-E, (C) Relative expression of genes from starch pathways in CMO-X and (D) RPKM values of genes from starch pathways in CMO-X. Results are the mean values from three independent experiments. Vertical bars represent SD. 
Table S3. Sugar contents during different developmental stages of C. moschata fruit.

\begin{tabular}{|c|c|c|c|c|c|c|}
\hline Days & \multicolumn{2}{|c|}{ Fructose (mg/g DW) } & \multicolumn{2}{c|}{ Glucose (mg/g DW) } & \multicolumn{2}{c|}{ Sucrose (mg/g DW) } \\
\hline & CMO-E & CMO-X & CMO-E & CMO-X & CMO-E & CMO-X \\
\hline 0 & $49.88 \pm 1.76$ & $51.06 \pm 1.64$ & $89.86 \pm 3.15$ & $57.48 \pm 2.90$ & $10.88 \pm 1.86$ & $7.9 \pm 0.36$ \\
\hline 5 & $93.04 \pm 2.88$ & $85.8 \pm 4.19$ & $108.39 \pm 3.01$ & $60.58 \pm 7.06$ & $11.75 \pm 1.32$ & $8.77 \pm 0.03$ \\
\hline 10 & $105.9 \pm 4.13$ & $84.89 \pm 5.26$ & $139.97 \pm 7.23$ & $70.35 \pm 5.91$ & $19.13 \pm 1.05$ & $14.04 \pm 0.01$ \\
\hline 20 & $107.4 \pm 8.81$ & $103.72 \pm 11.43$ & $154.77 \pm 7.50$ & $75.53 \pm 6.19$ & $38.28 \pm 2.14$ & $29.73 \pm 0.24$ \\
\hline 30 & $102.6 \pm 6.08$ & $38.44 \pm 6.03$ & $153.94 \pm 6.52$ & $29.51 \pm 4.09$ & $115.46 \pm 10.96$ & $165.65 \pm 11.12$ \\
\hline 40 & $75.59 \pm 4.49$ & $31.52 \pm 3.00$ & $133.92 \pm 5.26$ & $27.5 \pm 4.20$ & $165.2 \pm 9.59$ & $208.59 \pm 11.41$ \\
\hline
\end{tabular}

